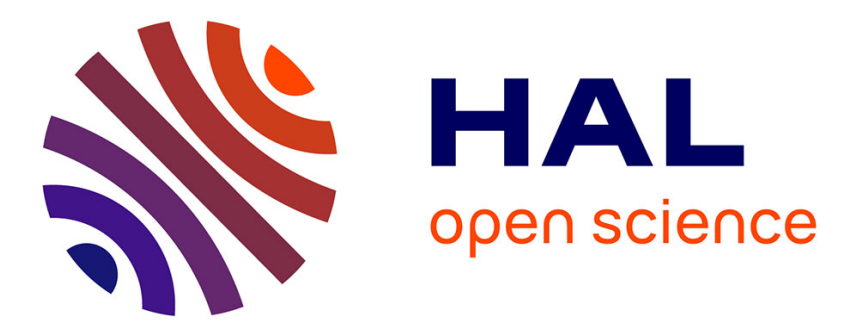

\title{
Novel Strategy for High Precision Automated Robotic Positioning based on Fabry-Perot Interferometry Principle
}

Houari Bettahar, Cédric Clevy, Florent Behague, Nadège Courjal, Philippe Lutz

\section{To cite this version:}

Houari Bettahar, Cédric Clevy, Florent Behague, Nadège Courjal, Philippe Lutz. Novel Strategy for High Precision Automated Robotic Positioning based on Fabry-Perot Interferometry Principle. International Conference on Automation Science and Engineering, Aug 2018, Munich, Germany. 10.1109/COASE.2018.8560700 . hal-02134422

\section{HAL Id: hal-02134422 \\ https://hal.science/hal-02134422}

Submitted on 20 May 2019

HAL is a multi-disciplinary open access archive for the deposit and dissemination of scientific research documents, whether they are published or not. The documents may come from teaching and research institutions in France or abroad, or from public or private research centers.
L'archive ouverte pluridisciplinaire HAL, est destinée au dépôt et à la diffusion de documents scientifiques de niveau recherche, publiés ou non, émanant des établissements d'enseignement et de recherche français ou étrangers, des laboratoires publics ou privés. 


\title{
Novel Strategy for High Precision Automated Robotic Positioning based on Fabry-Perot Interferometry Principle
}

\author{
Houari Bettahar ${ }^{1}$, Cédric Clévy ${ }^{1}$, Florent Behague ${ }^{2}$, Nadège Courjal $^{2}$ and Philippe Lutz ${ }^{1}$
}

\begin{abstract}
On account of the micro-scale building components manipulation and high precision demands, the interest is oriented toward automated robotic micro-manipulation and micro-assembly to provide low-cost, high performances, notably for integrated optical devices. The paper proposes a novel strategy for high precision fully automated robotic alignment. This strategy permits high accurate and fast automated alignment of two optical building structures (optical fiber, optical component) with optimal optical function in a known referencing between the robotic manipulator and the optical axis. The strategy allows to identify and to compensate the optical component misalignment angles and the robot translation error angles yielded from the robotic manipulator. The approach relies on robotic positioning combined with the use of FabryPerot interferometry of the reflected light irradiance for closed loop control. Fabry-Perot interference principle is especially used to give a rapid and high precision measurement. A photo-robotic positioning model is proposed that relates the optical component misalignment angles and robot translation error angles with the Fabry-Perot measurements. A 6 Degree-Of- Freedom (DOF) robotic platform is used to relatively align an optical component to an optical fiber for experimental validation. The obtained results leads to robotic positioning uncertainty of about $0.0021^{\circ}$ and alignment time of less than 12 s.
\end{abstract}

\section{INTRODUCTION}

Micro-robotics is very interesting for many industrial and biomedical fields, to assemble hybrid miniaturized systems, notably integrated optical systems.

The increasing functionality and complexity of integrated optical systems requires the integration of various building components fabricated with different technologies in order to achieve unique microsystems. These different fabrication technologies utilize different processes or materials. Hence, micro-assembly can bring very relevant solutions to overcome the monolithical integration diffuculties and to achieve 3D multifunctional optical devices fabricated with different technologies [1], [2], [3]. Moreover, microassembly permits

${ }^{1}$ H.Bettahar, ${ }^{1}$ C.Clévy, ${ }^{1}$ P.Lutz are with FEMTO-ST Institute, AS2M department, Univ. Bourgogne FrancheComté, UFC/CNRS/ENSMM, 24 rue Savary, 25000 Besançon, France, (e-mails: houari.bettahar@femto-st.fr, cclevy@femto-st.fr, philippe.lutz@femto-st.fr

${ }^{2}$ F.Behague, ${ }^{2}$ N.Courjal are with FEMTOST Institute, Optics department, Univ. Bourgogne Franche-Comté, UFC/CNRS/ENSMM, (e-mails:florent.behague@femto-st.fr, nadege.courjal@femto-st.fr) the development of complex integrated optical devices such as fast-axis-collimation [4], Fourier transform microspectrometers [5], etc.

On account of the characteristics of tiny building components manipulation and high precision demands, the interest is oriented toward automated robotic micro-manipulation and micro-assembly [6], [7]. This later will greatly increase the productivity and lower the cost of the assembled integrated optical devices. Therefore, full automation is a promising step towards commercial success in microassembly technology [8].

The microassembly in 3-dimensional (3-D) space especially with multi-degree-of-freedom (DOF) high accurate positioning remains open. There are passive approaches where optical elements are aligned by using passive alignment structures such as V-grooves [9] or patterned alignment marks [10]. This kind of approaches are quicker, costless and are limited to an accuracy of about $1 \mu \mathrm{m}$ [11], it is enough accurate for some dedicated specific applications as demonstrated for passive alignment of two photonic chips on a silicon optical bench [12].

There are other approaches use visual geometical feedback information to control the optical component poses [13], [14]. These approaches are more generic, they can reach $100 \mathrm{~nm}$ positioning accuracy [15] and about $1 \mu \mathrm{m}$ for complex 3D tasks [16]. However, geometrical positioning does not provide the optimal optical function. The last is the active approach, it is based on relative motions (motorized or not) to adjust the position of the optical components. The feedback control signal for the high-accurate multi-axis motion stage are provided from the transmitted power maximisation [17], [18], [19].

In previous works [20] we used an active alignment based on FP interferometry because FP (Fabry-Perot) cavity almost always happens when assembling integrated optical components (it then appears extremely generic), it also provides very high measurement quality (interferometric principle) but only provides displacement measurements along the optical axis (1D). Taking that specificities into account, the originality of [20] was to combine this FP interferometry feedback with robot nanopositioning : the nanopositionning robot enables to achieve motions useful to identify the position of the optical component relative to the optical fiber, then to automatically position the two components relatively at high speed to achieve high quality optical signal 
(irradiance i.e. light intensity). These works also show that remaining positioning errors, despite small, are likely due to the unknown referencing of the position of the robot joint motions relative to the optical components. This also may induce local maximum of the optical intensity only and likely limit the repeatability. For these reasons, the present paper targets at getting improved optical performances (better irradiance being correlated to better positioning accuracy). For that, the proposed approach consists in defining an original robot strategy able to define and consider the real robotic positioning frames in regard with the optical ones. As far as goes our knowledge, this lock has never been solved despite many interest drawn by active alignment techniques and high needs for assembled integrated optical components.

The paper is organized as follows. Section II explains the Fabry-Perot interferometry principle. Section III presents the proposed photo-robotic model for high accurate positioning with maximum irradiance. Section IV shows the experimental multi-DOF platform. Section $\mathrm{V}$ investigates and quantifies model performances and automated high positioning accuracy with experiments. Section VI concludes the paper.

\section{FABRY-PEROT MEASUREMENT PRINCIPLE}

The principle of light interferometry of light is utilized in various high precision measuring systems and sensors. The Fabry-Perot interferometry uses the phenomenon of multiple beam interference that arises when light shines through a cavity bounded by two reflective parallel surfaces (optical component and fiber ferrule). Each time the light encounters one of the surfaces, a portion of it is transmitted out, and the remaining part is reflected back. The net effect is to break a single beam into multiple beams which interfere with each other. The more reflections in the FabryPerot cavity, the more restrictive the constructive interferences will, leading to sharp resonance peaks. For this sake, the Fabry-Perot interferometry is used for relative misalignment and robot translation error angles identification. Specifically, the Fabry-Perot optical cavity between the end of a fiber ferrule and an optical component is exploited. The fiber is surrounded by a ferrule at its output, to ease manipulation. Therefore, in what follows we will talk about "fiber ferrule" to descibe the fiber output. The system acts like the Fabry-Perot interferometer. A fibered circulator is used to measure the reflected interference response as depicted in Fig. 1. When the optical component surface moves along $\vec{x}$, a change of the Fabry-Perot cavity length $(L)$ happens. Fig. 1 shows typical interferences when $L$ varies, the periodicity of the irradiance signal directly depends on the wavelength of the laser signal $(\lambda=1560$ in the case of Fig. 2), the cavity distance variation $\delta L$ needed to go from one resonance peak to the next one is multiple

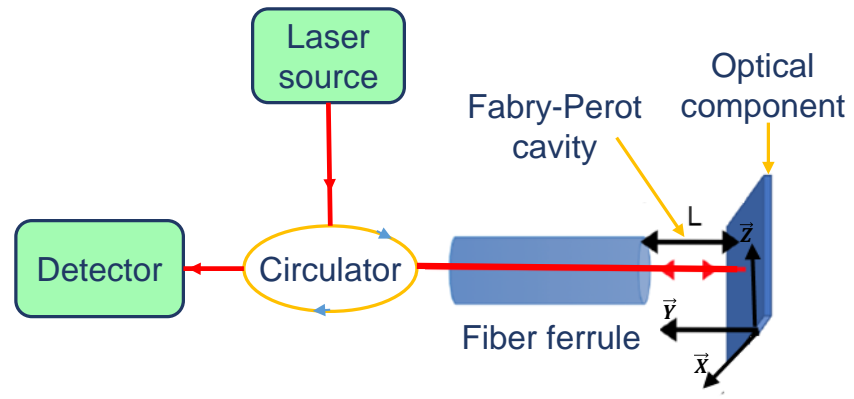

Fig. 1: Principle of measuring the reflected light from the system behaving as a Fabry-Perot interferometer.

of $\lambda / 2$. If $p$ is a natural number, the distance between two reasonances can be written as :

$$
\delta L=\frac{\lambda}{2} \cdot p
$$

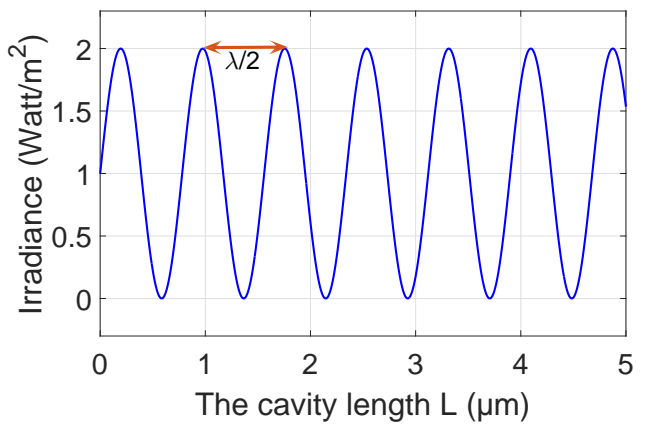

Fig. 2: Reflected irradiance versus Fabry-Perot cavity length $L$.

\section{PHOTO-ROBOTIC POSITIONING MODEL}

This section is divided into two subsections, the first will model the relative misalignment between the fiber ferrule and the optical component by defining a robot strategy able to define and consider the real robotic positioning frames in regard with the optical ones. The second subsection will discuss the angular alignment and irradiance maximization relationship.

\section{A. Opto-mechnical model based on relative misaligne- ment and robot translation error angles}

As shown in Fig. 3, the principle of Fabry-Perot measurement is used to accurately identify angular misalignments. Two optical components are used for angular positioning. The fiber ferrule is chosen to be fixed. The optical component is fixed on a moving 6 DOF robotic manipulator, so it can move with 3 translations and 3 rotations with respect to the fiber ferrule. Thus, the idea is to compensate the relative 
angular misalignment between the ferrule and the optical component, and the influencing robot translations angular errors based on a known referencing.

The frame $\left(O_{G} Y_{G} Z_{G}\right)$ is assigned to the end-effector robotic manipulator, the origin $O_{G}$ is assigned to its real rotational center, the $\overrightarrow{O_{G} Y_{G}}$ and $\overrightarrow{O_{G} Z_{G}}$ are the real motion directions of the end-effector. The frame $\left(\begin{array}{llll}O_{L} & Y_{L} & Z_{L}\end{array}\right)$ is assigned to the optical component, its origin is assigned to its outer surface center, the vector $\overrightarrow{O_{L} Y_{L}}$ is chosen to be perpendicular to the outer optical component surface and then $\overrightarrow{O_{L} Z_{L}}$ is perpendicular to $\overrightarrow{O_{L} Y_{L}}$. The frame $\left(O_{F} Y_{F} Z_{F}\right)$ is assigned to the fiber ferrule, its origin $O_{F}$ is assigned to the intersection point between the optical axis and the outer plane of the optical component, $\overrightarrow{O_{F} Y_{F}}$ is carried by the optical axis and it is directed toward the optical component, $\overrightarrow{O_{F} Z_{F}}$ is perpendicular to $\overrightarrow{O_{F} Y_{F}}$.

The previously described principle is used to accurately identify the relative misalignments and robot translations angular errors $\left(\beta_{i}, \theta_{i}\right)$ and $\left(\beta_{l}, \theta_{l}\right)$ respectively. Indeed, if the optical component has initially a $\beta_{i}$ fault angle (see Fig. 3), then a scan of the along the $Z_{G}$-axis with a known scan displacement $L_{z}$ is expected to induce a continuous cavity distance variation $L_{y}$. Consequently, it generates an oscillating reflected light irradiance at the same time. From the number of maxima of this oscillating signal, the propagated distance $L_{y}$ can be evaluated using equation (1) and as shown in Fig. 2. From the twice assessment of the distance $L_{y}$, and for a given vertical scan distance $L_{z}$, the angle $\beta_{i}$ and $\beta_{l}$ can be calculated using equation 2 .

$$
\beta_{i}=\arctan \left(\frac{L_{y}-L_{z} \sin \left(\beta_{l}\right)}{L_{z} \cos \left(\beta_{l}\right)}\right)
$$

Where $\beta_{i}$ is the angle between the surface of the optical component and the $Z_{F}$-axis in the $\left(X_{G} O_{G} Y_{G}\right)$ plane and $\beta_{l}$ is the angle between $Z_{F^{-}}$axis and the $Z_{G^{-}}$ axis in the $\left(X_{G} O_{G} Y_{G}\right)$ plane.

$$
\begin{aligned}
& \beta_{=} \arctan \left(\frac{L_{y}}{L_{z}^{\prime}}\right) \\
& L_{z}^{\prime}=L_{z} \cos \left(\beta_{l}\right)
\end{aligned}
$$

Once the robot translation angular error $\beta_{l}$ is identified and compensated using equation (4), where $L_{z}^{\prime}$ is the projection of $L_{z}$ on the perpendicular axis $\left(O_{L} Z_{L}\right)$. Then equation (3) can be used for the identification of the $\beta_{i}$ with only one scan $L_{z}$ for each relative misalignment angle.

The same steps are followed as previously mentioned for $\theta_{i}$ and $\theta_{l}$ relative misalignment and robot translation error angles, where $\theta_{i}$ is the angle between the surface of the optical component and the $X_{F}$-axis in the $\left(Z_{G} O_{G} Y_{G}\right)$ plane and $\theta_{l}$ is the angle between $X_{F}$-axis and the $X_{G}$-axis in the $\left(Z_{G} O_{G} Y_{G}\right)$ plane.

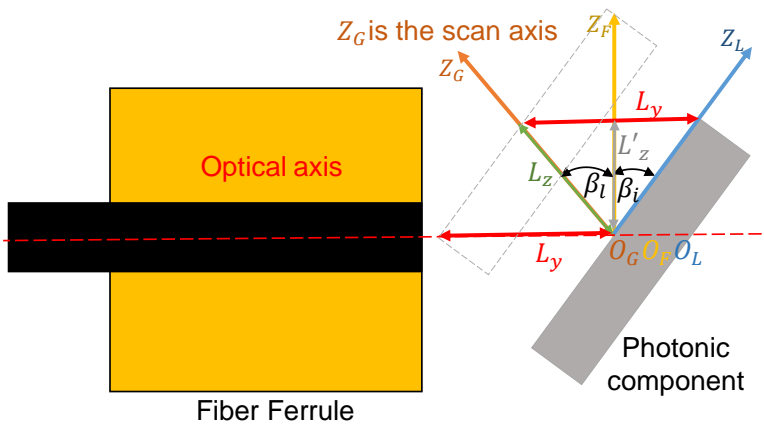

Fig. 3: Relative misalignment angle $\beta_{i}$ and robot translation error angle $\beta_{l}$ identification based on FabryPerot measure and optical component scan. $\beta_{i}$ is the angle between the surface of the optical component and the $Z_{F}$-axis in the $\left(X_{G} O_{G} Y_{G}\right)$ plane. $\beta_{l}$ is the angle between $Z_{F}$-axis and the $Z_{G}$-axis in the $\left(X_{G} O_{G} Y_{G}\right)$ plane.

\section{B. Angular alignment and irradiance maximization re- lationship}

After having integrated robot translation error angles, $\overrightarrow{O_{G} Y_{S}}$ axis is the new robot motion axis, it is parallel to the optical axis and directed toward the fiber ferrule. Moving the optical component with no relative angular misalignment continuously toward the fiber in $Y_{S}$ direction for a certain distance (see Fig. 4), it yields the maximum oscillating reflected light amplitude. On the other hand, moving the optical component with some relative angular misalignment the same as for the first time, it yields an oscillating reflected light with reduced amplitude. The real rotational center of the goniometer $O_{G}$ is far away from the optical component center $O_{L}$ as shown in Fig. 4. After having identified angular misalignment using the described method above, the correction step will induce a simultaneous displacement of optical component on $Y_{S}$ direction. So after correction, the alignment is guaranteed but the maximum irradiance isn't guaranteed since there are many maxima and minima along this displacement as shown in Fig. 5. As a conclusion, controlling this displacement distance and knowing its exact irradiance maxima placement as well can guarantee an accurate alignment with maximum irradiance.

\section{EXPERIMENT PLATFORM}

The experimental set up proposed for automated relative positioning of the optical component with respect to the fiber ferrule as shown in Fig. 7. A 6 DOF robotic micro-manipulator is proposed to position the optical component and control its poses. An other XYZ stage is fixed in front of the 6 DOF micro-manipulator is used to hold the fiber ferrule.

The 6 DOF robotic micro-manipulator comprises P563 PIMars Nano-positioning stage for the translational motion on $\mathrm{X}, \mathrm{Y}, \mathrm{Z}$ directions, the displacement 


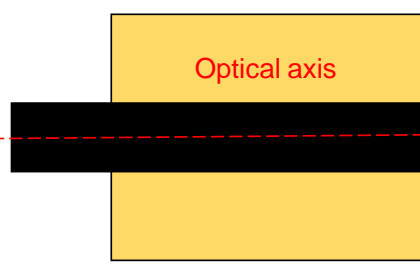

Fiber Ferrule

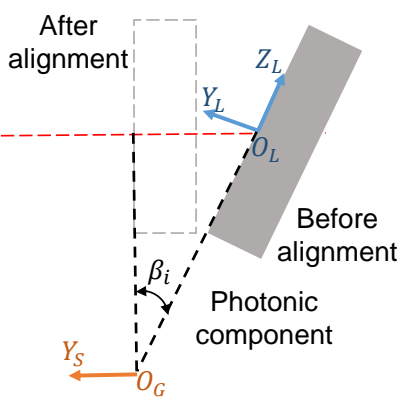

Fig. 4: The yielded displacement during $\beta_{i}$ relative misalignment correction, when the end-effector rotational center is far away from the outer surface center of the optical component.

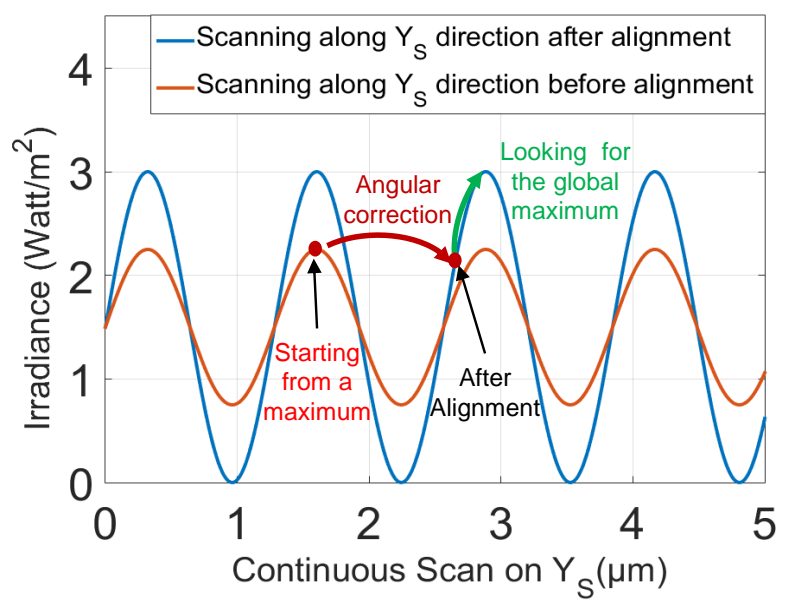

Fig. 5: Angular positioning and irradiance maximization relationship.

range of each translation is $300 \mu \mathrm{m}$. SGO-60.5 and SGO-77.5 goniometers are used for rotational motion around $\mathrm{Z}$-axis and $\mathrm{X}$-axis respectively, each having a rotating range of $10^{\circ}$. A rotary positioner is used for rotation around $\mathrm{Y}$-axis, and its rotating range is $360^{\circ}$. Fig. 6 and Fig. 10, show the kinematic model and the experimental platform.

The optical set up consists of a laser light source (wavelength $\lambda=1560 \mathrm{~nm}$ ) connected to a circulator and then to the fiber ferrule. This configuration permits to align and assemble the optical component to the fiber. The reflected irradiance from optical component through the fiber ferrule is the closed loop information for the robotic micro-manipulator poses control.

This set up permits the acquisition of irradiance signal from the reflected light from the optical component surface, returning back through the fiber ferrule and then through the circulator. Fabry-Perot measurement is used then to control the relative position between fiber ferrule/optical component actively.

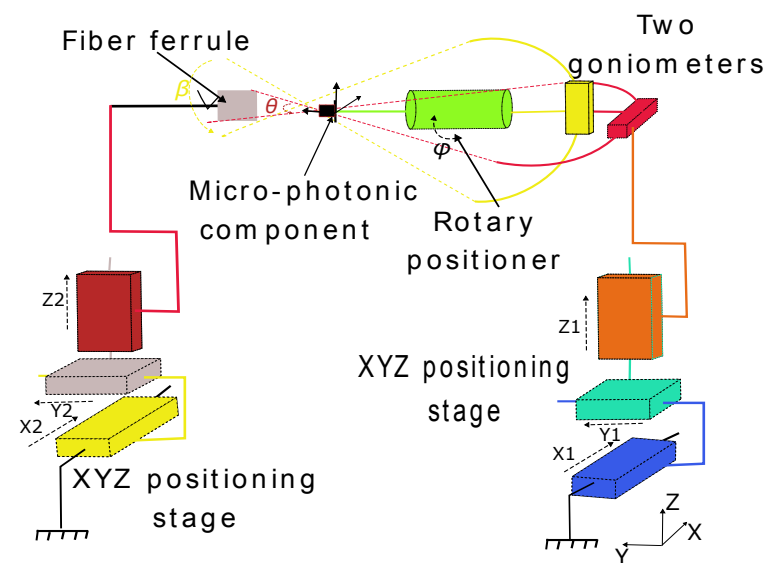

Fig. 6: The kinematic scheme of the proposed robotic micro-manipulator for micro-alignment of microoptical components.

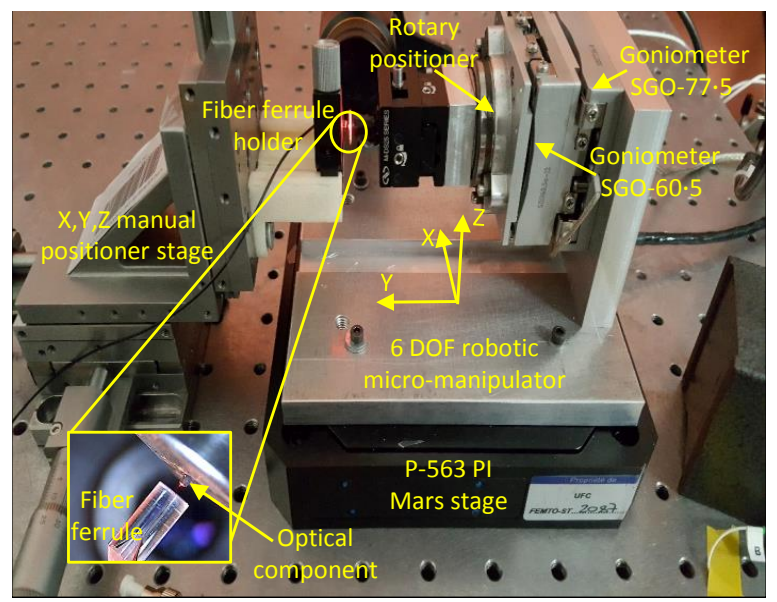

Fig. 7: The experimental set up workstation.

\section{POSITIONING STRATEGY AND EXPERIMENTAL VALIDATION}

In this section, the relative misalignment and the robot translation error angles identification based on Fabry-Perot interferometry principle presented in Section III is implemented. The experimental set-up and the photo-robotic scheme presented in Section V are realized using Matlab/Simulink platform.

The experiments were realized within a constant environmental conditions $\left(\mathrm{T}=20^{\circ} \mathrm{C}\right)$.

In order to investigate the identified angles repeatability, 20 different angular configurations were given for the optical component with a constant $\left(\delta \beta_{g}=0.25^{\circ}\right)$ steps between each configuration. $\beta_{g}$ is the robotic manipulator angles rotating around $O_{G} X_{G}$ axis. A thirty scan along $Z_{G}$-axis were done for each configuration. Then from each two configurations the the relative misalignment and therobot translation error angles $\beta_{i}$ and $\beta_{l}$ were identified. The two angles were identified 30 times.

The same steps are followed for $\theta_{i}$ and $\theta_{l}$ relative misalignment and robot translation error angles, where 
$\theta_{g}$ is the robotic manipulator angles rotating around $O_{G} Z_{G}$ axis. The obtained results are presented in Table I. Table I shows the obtained results for average values, minimum values, maximum values and standard deviation.

Once the robot translation error angles are compensated, then the relative misalignment can be identified. Fig. 8 shows the reflected light irradiance before and after compensation for $\beta_{i}$ and $\theta_{i}$ relative misalignment for a circular scan. Applying a circular scan, yields FabryPerot cavity variation during the scan as represented by the peaks appearance as shown in Fig. 8. After compensation and by applying a verification circular scan, we always obtain a global maximum constant irradiance, which means that there is no Fabry-Perot cavity variation during the scan.

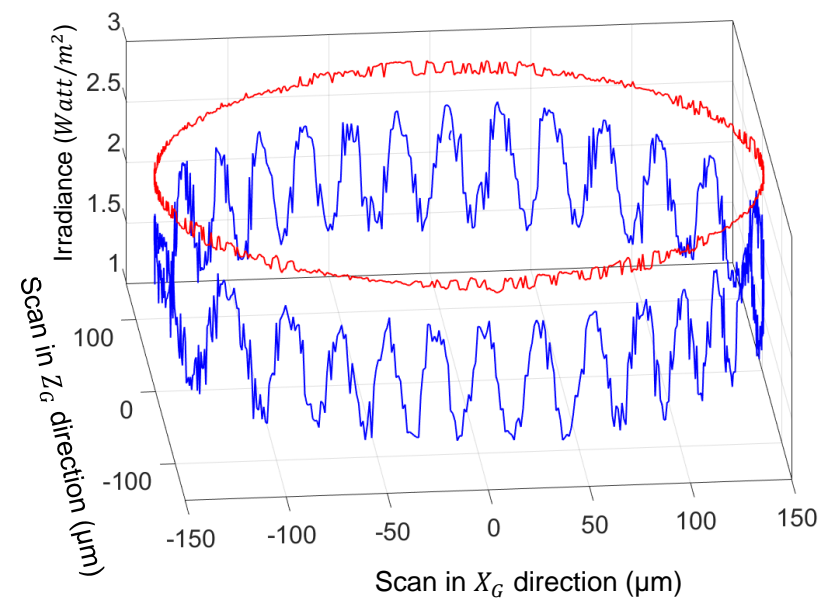

Fig. 8: The reflected light irradiance before (blue) and after (red) correction for $\beta_{i}$ and $\theta_{i}$ misalignment for a circular scan.

After having identified the relative misalignment angles, the next step is to correct these relative misalignments. For this sake, the relationship between the end-effector angles $\left(\beta_{g}, \theta_{g}\right)$ and the corresponding identified angles $\left(\beta_{i}\right.$ and $\left.\theta_{i}\right)$ has to be determined. Different angular configurations were given to the optical component around $Z_{G}$ and then around $X_{G}$ with a constant steps $\left(\delta \beta_{g}=0.25^{\circ}, \delta \theta_{g}=0.25^{\circ}\right)$ respectively between each configuration. A 30 times identification process as performed for each configuration. Fig. 9 and Fig. 11 shows the linear relationship between the identified and the end-effector angles.

Fig. 10 and Fig. 12 shows the uncertainty distribution of the identified relative misalignment angles $\beta_{i}$ and $\theta_{i}$ respectively for each robotic end-effector angle. Based on the obtained results, the uncertainty increases when the identified peaks number is less than 5 (peaks number $\leq 5$ ) for $\beta_{i}$ angles, and the uncertainty increases when the identified peaks number is less than 7 (peaks number $\leq 7$ ) for $\theta_{i}$ angles.

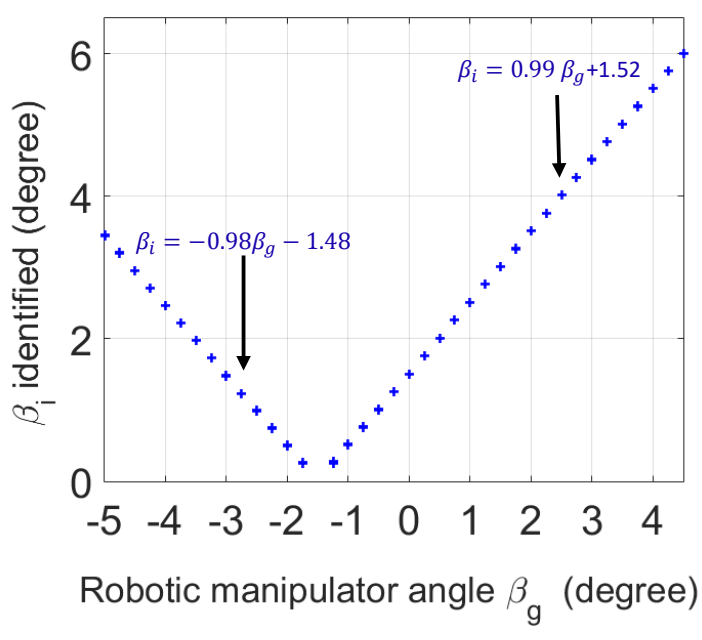

Fig. 9: The identified angle $\beta_{i}$ (output) and the endeffector robotic manipulator angle $\beta_{g}$ (input) linear relationship.

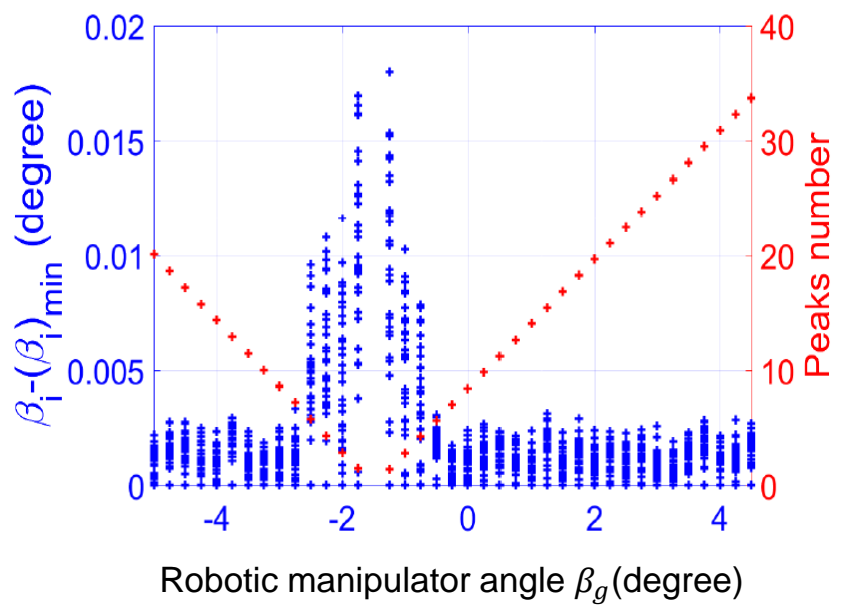

Fig. 10: The $\beta_{i}$ identified angle uncertainty illustration for each end-effector angle.

Thanks to this uncertainty analysis which allows us to perform automated high accurate alignment with very small uncertainty. By taking relative misalignment angles having the corresponding peaks number greater than 7 ((peaks number $\geq 7)$ ) permits to avoid the high uncertainty zone. Unlike the classical active alignment, they use iterative (step-by-step) irradiance maximisation, where they converge systematically toward the high uncertainty zone, it introduces local maximum results with more uncertainty and errors. The obtained uncertainty result for the proposed strategy based on standard deviation is $0.002^{\circ}$.

Finally based on the above studies, the approved automated high accurate alignment with very small uncertainty flowchart is shown in Fig. 13. Where all alignment process is realized in less then $12 \mathrm{~s}$. 
TABLE I: The identified robot translation error and relative misalignment angles values

\begin{tabular}{ccccc}
\hline \hline Angles & Mean value & maximum value & minimum value & standard deviation \\
\hline$\beta_{l}$ (degree) & 0.7453 & 0.7517 & 0.7436 & 0.0021 \\
$\theta_{l}$ (degree) & 0.8415 & 0.8512 & 0.8387 & 0.0032 \\
$\beta_{i}$ (degree) & 1.7538 & 1.7602 & 1.7507 & 0.0021 \\
$\theta_{i}($ degree $)$ & 1.8523 & 1.8612 & 1.8481 & 0.0032 \\
\hline \hline
\end{tabular}

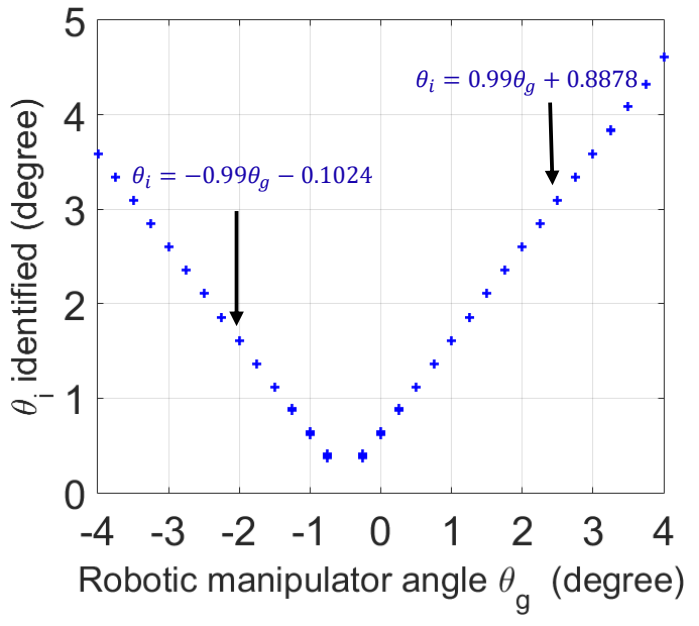

Fig. 11: The identified angle $\theta_{i}$ (output)and the endeffector robotic manipulator angle $\theta_{g}$ (input) linear relationship.

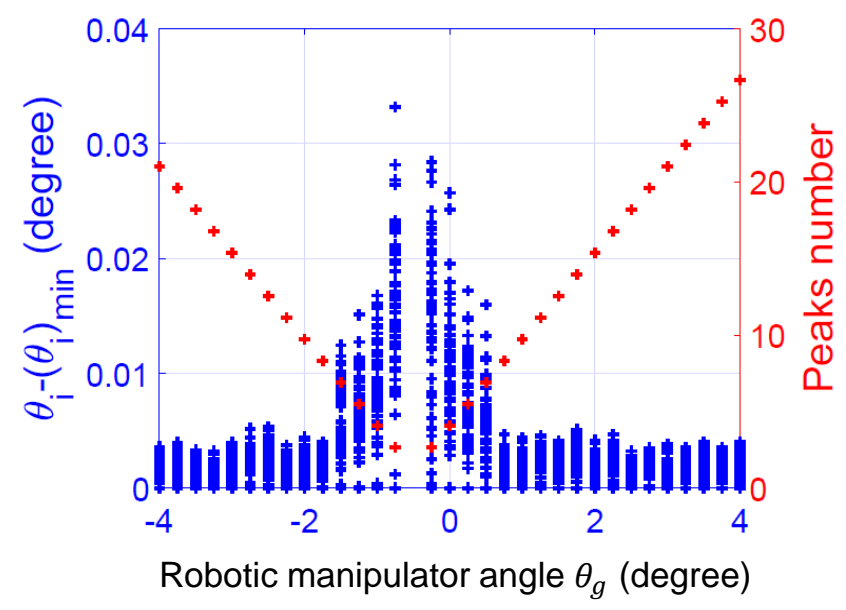

Fig. 12: The $\theta_{i}$ identified angle uncertainty illustration for each end-effector angle.

\section{CONCLUSION}

Recent development in integrated optical devices require advances in the optical positioning and assembly of the building components. The high need to

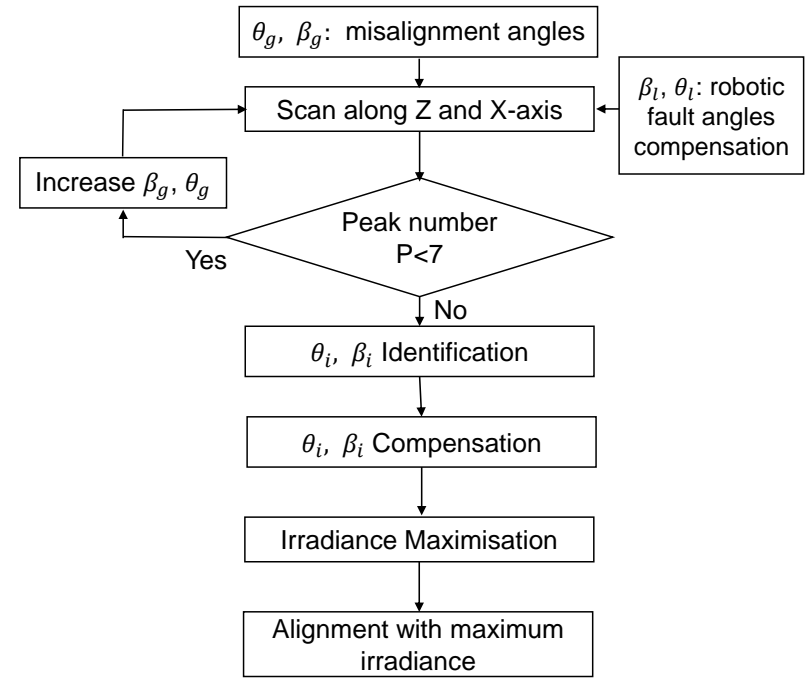

Fig. 13: Automated high accurate alignment with very small uncertainty flowchart.

increase the task speed and also repeatability leads to robotic assembly which is less used (manual approach widespread). In the paper we used an optical phenomenon that almost always happens during such assembly (Fabry-Perot). The contribution of the paper lies in the robot strategy able to define and consider the real robotic positioning frames in regard with the optical ones in an automated, systematic and online way.

The paper deals with an integrated optics system accomplished through high accurate multi-DOF robotic active positioning strategy. For this sake, two structural optical components were chosen, a fiber ferrule and an optical component. Fabry-Perot interferometry principle was used to provide a rapid and high accurate measure. the same principle was used for accurate misalignment and robot translation error angles identification. A photo-robotic positioning model consists in defining a robot strategy able to define and consider the real robotic positioning frames in regard with the optical ones. It permits to provide an optimal optical positioning function and accurate angular identification and compensation in fast time (less than $12 \mathrm{~s}$ ). Using 
the proposed model and the uncertainty quantification, the automated angular misalignment measurements and compensation were realized with a standard deviation of $0.0021^{\circ}$.

\section{ACKNOWLEDGMENT}

These works have been funded by the Franche-Comté region, nano-roboptic project supported by the Labex ACTION project, ANR CEPAGE ANR-16-CE240024, ANR COLAMIR ANR-16-CE10-0009 and by the French RENATECH network through its FEMTO-ST technological facility.

\section{REFERENCES}

[1] J. Agnus, N. Chaillet, C. Clévy, S. Dembélé, M. Gauthier, Y. Haddab, G. Laurent, P. Lutz, N. Piat, K. Rabenorosoa et al., "Robotic microassembly and micromanipulation at femto-st," Journal of Micro-Bio Robotics, vol. 8, no. 2, pp. 91-106, 2013.

[2] L. Zimmermann, G. B. Preve, T. Tekin, T. Rosin, and K. Landles, "Packaging and assembly for integrated photonics-a review of the epixpack photonics packaging platform," IEEE Journal of Selected Topics in Quantum Electronics, vol. 17, no. 3, pp. 645-651, 2011.

[3] O. Benson, "Assembly of hybrid photonic architectures from nanophotonic constituents," Nature, vol. 480, no. 7376, pp. 193-199, 2011.

[4] J. Miesner, A. Timmermann, J. Meinschien, B. Neumann, S. Wright, T. Tekin, H. Schröder, T. Westphalen, and F. Frischkorn, "Automated assembly of fast-axis collimation (fac) lenses for diode laser bar modules," in SPIE LASE : Lasers and Applications in Science and Engineering. International Society for Optics and Photonics, 2009, pp. 71980G71 980G.

[5] A. N. Das, D. O. Popa, J. Sin, and H. E. Stephanou, "Precision alignment and assembly of a fourier transform microspectrometer," Journal of Micro-Nano Mechatronics, vol. 5, no. 1-2, p. 15, 2009.

[6] G. Böttger, D. Weber, F. Scholz, H. Schröder, M. SchneiderRamelow, and K.-D. Lang, "Fully automated hybrid diode laser assembly using high precision active alignment," in SPIE LASE. International Society for Optics and Photonics, 2016, pp. $97300 \mathrm{E}-97300 \mathrm{E}$.

[7] B. Komati, K. Rabenorosoa, C. Clévy, and P. Lutz, "Automated guiding task of a flexible micropart using a twosensing-finger microgripper," IEEE Transactions on Automation Science and Engineering, vol. 10, no. 3, pp. 515-524, 2013.

[8] C. Brecher, N. Pyschny, S. Haag, and V. G. Lule, "Micromanipulators for a flexible automated assembly of micro optics," in SPIE Photonics Europe. International Society for Optics and Photonics, 2012, pp. 84 280J-84 280J.

[9] R. Takigawa, E. Higurashi, T. Suga, and T. Kawanishi, "Passive alignment and mounting of linbo _ 3 waveguide chips on si substrates by low-temperature solid-state bonding of au," IEEE Journal of Selected Topics in Quantum Electronics, vol. 17, no. 3, pp. 652-658, 2011.

[10] R. Hauffe, U. Siebel, K. Petermann, R. Moosburger, J.R. Kropp, and F. Arndt, "Methods for passive fiber chip coupling of integrated optical devices," in Electronic Components \& Technology Conference, 2000. 2000 Proceedings. 50th. IEEE, 2000, pp. 238-243.

[11] B. Li, H. Wirz, and A. Sharon, "Optimizing fiber coupling with a quasi-passive microoptical bench," Journal of, Microelectromechanical Systems, vol. 14, no. 6, pp. 1339-1346, 2005.

[12] J. F. C. van Gurp, M. Tichem, U. Staufer, and J. Zhao, "Passive photonic alignment with submicrometer repeatability and accuracy," Components, Packaging and Manufacturing Technology, IEEE Transactions on, vol. 3, no. 11, pp. 19711979, 2013.
[13] A. Kudryavtsev, G. J. Laurent, C. Clévy, B. Tamadazte, and P. Lutz, "Analysis of cad model-based visual tracking for microassembly using a new block set for matlab/simulink," International Journal of Optomechatronics, vol. 9, no. 4, pp. 295-309, 2015.

[14] Y. Zheng, X.-c. Kai, J.-a. Duan, and B.-b. Li, "Automated visual position detection and adjustment for optical waveguide chips and optical fiber arrays," Journal of Central South University, vol. 22, pp. 3868-3875, 2015.

[15] Z. Chen, D. Zhou, H. Liao, and X. Zhang, "Precision alignment of optical fibers based on telecentric stereo microvision," IEEE/ASME Transactions on Mechatronics, vol. 21, no. 4, pp. 1924-1934, 2016.

[16] C. Clévy, I. Lungu, K. Rabenorosoa, and P. Lutz, "Positioning accuracy characterization of assembled microscale components for micro-optical benches," Assembly Automation, vol. 34, no. 1, pp. 69-77, 2014.

[17] R. Zhang and F. G. Shi, "A novel algorithm for fiberoptic alignment automation," Advanced Packaging, IEEE Transactions on, vol. 27, no. 1, pp. 173-178, 2004.

[18] M. Landry, A. Kaddouri, Y. Bouslimani, and M. Ghribi, "Automated alignment of microstructured optical fibers and conventional single-mode fibers," Optical Engineering, vol. 52, no. 6, pp. 065003-065003, 2013.

[19] C.-Y. Tseng and J.-P. Wang, "Automation of multi-degreeof-freedom fiber-optic alignment using a modified simplex method," International Journal of Machine Tools and Manufacture, vol. 45, no. 10, pp. 1109-1119, 2005.

[20] H. Bettahar, A. Caspar, C. Clévy, N. Courjal, and P. Lutz, "Photo-robotic positioning for integrated optics," IEEE Robotics and Automation Letters, vol. 2, no. 1, pp. 217-222, 2017. 